\title{
Maternal dietary quality, inflammatory potential and offspring adiposity throughout childhood: a pooled analysis of 7 European cohorts (ALPHABET consortium)
}

\author{
Ling-Wei Chen $^{1}$, Adrien Aubert ${ }^{2}$, Jonathan Y. Bernard ${ }^{2}$, Cyrus Cooper $^{3}$, Liesbeth Duijts ${ }^{4}$, \\ Aisling A. Geraghty ${ }^{5}$, Nicholas C. Harvey ${ }^{3}$, James R. Hebert ${ }^{6}$, Barbara Heude ${ }^{2}$, \\ Cecily C. Kelleher ${ }^{1}$, Fionnuala M. McAuliffe ${ }^{5}$, John Mehegan ${ }^{1}$, Rosalie Mensink-Bout ${ }^{4}$, \\ Kinga Polanska ${ }^{7}$, Caroline L. Relton ${ }^{8}$, Nitin Shivappa ${ }^{6}$, Matthew Suderman ${ }^{8}$ and \\ Catherine M Phillips ${ }^{1}$ \\ ${ }^{1}$ HRB Centre for Diet and Health Research, School of Public Health, Physiotherapy, and Sports Science, University \\ College Dublin, Dublin, Ireland, \\ ${ }^{2}$ Centre for Research in Epidemiology and Statistics (CRESS), INSERM, Villejuif, France, \\ ${ }^{3}$ MRC Lifecourse Epidemiology Unit (University of Southampton) Southampton General Hospital Southampton, \\ Southampton, United Kingdom, \\ ${ }^{4}$ Department of Pediatrics, Erasmus MC, University Medical Center Rotterdam, Rotterdam, Netherlands, \\ ${ }^{5}$ UCD Perinatal Research Centre, School of Medicine, University College Dublin, National Maternity Hospital, \\ Dublin, Ireland, \\ ${ }^{6}$ Arnold School of Public Health, University of South Carolina, Columbia, South Carolina, USA, \\ ${ }^{7}$ Nofer Institute of Occupational Medicine, Lodz, Poland and \\ ${ }^{8}$ MRC Integrative Epidemiology Unit, University of Bristol, Bristol, United Kingdom
}

\begin{abstract}
Introduction: The foetal programming hypothesis posits that optimising early life factors e.g. maternal diets can help avert the burden of adverse childhood outcomes e.g. childhood obesity. To improve applicability to public health messaging, we investigated whether maternal whole diet quality and inflammatory potential influence childhood adiposity in a large consortium.

Methods: We harmonized and pooled individual participant data from up to 8,769 mother-child pairs in 7 European mother-offspring cohorts. Maternal early-, late-, and whole-pregnancy dietary quality and inflammatory potential were assessed with Dietary Approaches to Stop Hypertension (DASH) and energy-adjusted Dietary Inflammatory Index (E-DII), respectively. Primary outcome was childhood overweight and obesity (OWOB), defined as age- and sex-specific body-mass-index-z score $(\mathrm{BMIz})>85^{\text {th }}$ percentile based on WHO growth standard. Secondary outcomes were sum-of-skinfold-thickness (SST), fat-mass-index (FMI) and fat-free-mass-index (FFMI) in available cohorts. Outcomes were assessed in early- [mean (SD) age: 2.8 (0.3) y], mid- [6.2 (0.6) y], and late-childhood [10.6 (1.2) y]. We used multivariable regression analyses to assess the associations of maternal E-DII and DASH with offspring adiposity outcomes in cohort-specific analyses, with subsequent random-effects meta-analyses. Analyses were adjusted for maternal age, pre-pregnancy BMI, parity, lifestyle factors, energy intake, educational attainment, offspring age and sex.
\end{abstract}

Results: A more pro-inflammatory maternal diet, indicated by higher E-DII, was associated with a higher risk of offspring late-childhood OWOB [pooled-OR $(95 \%$ CI) comparing highest vs. lowest E-DII quartiles: $1.22(1.01,1.47)$ for whole-pregnancy and $1.38(1.05,1.83)$ for early-pregnancy; both $P<0.05]$. Moreover, higher late-pregnancy E-DII was associated with higher mid-childhood FMI [pooled- $\beta(95 \%$ CI): $\left.0.11(0.003,0.22) \mathrm{kg} / \mathrm{m}^{2} ; P<0.05\right]$; trending association was observed for whole-pregnancy E-DII $\left[0.12(-0.01,0.25) \mathrm{kg} / \mathrm{m}^{2} ; P=0.07\right]$. A higher maternal dietary quality, indicated by higher DASH score, showed a trending inverse association with late-childhood OWOB (pooled-OR $(95 \% \mathrm{CI})$ comparing highest vs. lowest DASH quartiles: $0.58(0.32,1.02 ; P=0.06)$. Higher early-pregnancy DASH was associated with lower late-childhood SST [pooled- $\beta(95 \% \mathrm{CI}):-1.9(-3.6,-0.1) \mathrm{cm} ; P<0.05$ ] and tended to be associated with lower latechildhood FMI $\left[-0.34(-0.71,0.04) \mathrm{kg} / \mathrm{m}^{2} ; P=0.08\right]$. Higher whole-pregnancy DASH tended to associate with lower early-childhood SST $[-0.33(-0.72,0.06) \mathrm{cm} ; P=0.10]$. Results were similar when modelling DASH and E-DII continuously.

Discussion: Analysis of pooled data suggests that pro-inflammatory, low-quality maternal antenatal diets may influence offspring body composition and obesity risk, especially during mid- or late-childhood. Due to variation of data availability at each timepoint, our results should be interpreted with caution. Because most associations were observed at mid-childhood or later, future studies will benefit from a longer follow-up.

\section{Conflict of Interest}

There is no conflict of interest 